\title{
La impronta de Pierre Teilhard de Chardin en las tesis evolutivas de Karl Schmitz-Moormann
}

The imprint of Pierre Teilhard de Chardin on the evolutionary theses of Karl Schmitz-Moormann

\section{RICARD CASADESÚS}

Seminari de Teologia i Ciències de Barcelona, Institut de Teologia Fonamental, Sant Cugat del Vallès, España ricard.casadesus@gmail.com ORCID: 0000-0001-6758-2920

Resumen. En este artículo exponemos las intuiciones del teólogo y biólogo alemán Karl Schmitz-Moormann sobre la evolución. Como leitmotiv subyacente en toda su obra, Schmitz-Moormann tiene a Teilhard de Chardin. Inspirado por Teilhard y su metafísica de la unión, introduce un nuevo concepto, a saber, la uni-totalidad. Veremos aquí cómo Schmitz-Moormann observa diversas uni-totalidades a lo largo del progreso evolutivo, desde las partículas subatómicas hasta las agrupaciones humanas. Esas diversas uni-totalidades implican, a la vez, diversidad de unión, que puede ser explicada por causas que van desde la interacción nuclear hasta el amor interpersonal. Análogamente, Schmitz-Moormann ve nuestro Dios tri-uno como la Uni-totalidad suprema unida por amor, a la que se acercan las uni-totalidades creadas.

Palabras clave: metafísica de la unión; creación por unión; complejidad; unitotalidad; conciencia; Teilhard de Chardin. 
Abstract. In this paper we focus on the insights on evolution by the German biologist and theologian Karl Schmitz-Moormann. As underlying leitmotiv throughout all his work, Schmitz-Moormann has Teilhard de Chardin. Inspired by Teilhard and his metaphysics of union, Schmitz-Moormann introduces a new concept, that is uni-totality. This paper shows how Schmitz-Moormann sees various uni-totalities in the course of the process of evolution beginning from sub-atomic particles to human groups. These different uni-totalities also imply a diversity of union, that can be explained by causes which goes from nuclear interaction to interpersonal love. He analogously considers the triune God as the supreme Uni-totality united by love, to which created uni-totalities approximate.

Keywords: Metaphysics of union; creation by union; complexity; uni-totality; consciousness; Teilhard de Chardin.

\section{Introducción}

En la segunda mitad del s. XX, el biólogo y teólogo católico alemán Karl Schmitz-Moormann (1928-1996) reflexionó sobre la evolución cósmica y biológica a partir de la nueva concepción metafísica que la realidad de esa evolución impone, especialmente la idea de que el ser se va enriqueciendo por unión.

El pensamiento y las obras de Karl Schmitz-Moormann están directamente influidos por Pierre Teilhard de Chardin. Su interés científico y teológico por Teilhard de Chardin le llevó a editar en 1971, en colaboración con su mujer Nicole, los once volúmenes de la Obra Científica (crf. Teilhard de Chardin 1971) completa de Pierre Teilhard de Chardin, y en 1974-1977 los tres volúmenes de su Diario (cfr. Teilhard de Chardin 1974-1977) personal.

Pero antes de elaborar sus tesis evolutivas, Schmitz-Moormann se planteó unas cuestiones, a partir de argumentos teóricos y metodológicos, que contrastó con la observación de la realidad. Esto le llevó a establecer unos presupuestos previos a las tesis, que deben tenerse en cuenta. Estos presupuestos son dos: la falacia cuantitativa y la centralidad del ser humano en el universo.

\section{Falacia cuantitativa versus argumento cualitativo}

El progresivo conocimiento de la inmensidad del universo produce en los seres humanos una sensación de su propia insignificancia y pequeñez. 
Ciertamente, desde una perspectiva cuantitativa así es. El planeta Tierra es poco significativo en contraste con los miles de millones de galaxias del universo. Por tanto, cuantitativamente es correcto decir que la vida y, especialmente, los seres humanos son totalmente insignificantes en el universo.

Sin embargo, la cuestión que se pregunta Karl Schmitz-Moormann es si en la evolución del universo, la característica y el parámetro más significativo es la cantidad. Si se acepta la cantidad como parámetro para la evolución del universo, se llega a la conclusión paradójica de que la evolución constituye un fenómeno insignificante. En la siguiente figura (Fig. 1), se puede observar un conjunto de gráficos, que hemos elaborado, inspirados en el de Schmitz-Moormann ${ }^{1}$, en el que presentamos varias muestras dobles (95/5\%) de los sucesivos tipos de entidad que van evolucionando en el universo.

\begin{tabular}{|c||c|c|c|c|}
\hline $\begin{array}{c}\text { Radiación de fondo } \\
\text { Hidrógeno \& helio }\end{array}$ & $\begin{array}{c}\text { Hidrógeno\& helio } \\
\text { Resto de átomos }\end{array}$ & $\begin{array}{c}\text { Moléculas } \\
\text { Pacromoléculas }\end{array}$ \\
\hline $\begin{array}{c}\text { Procariotas } \\
\text { Eucariotas }\end{array}$ & Eucariotas Metazoos & Méculas \\
\hline
\end{tabular}

Fig. 1. Serie de muestras estadísticas $95 / 5 \%$ de varios tipos de entidad que evolucionan sucesivamene en otros más complejos

1 Schmitz-Moormann presenta un gráfico describiendo las funciones representadas en él como medias campanas de Gauss, pero, según nuestra opinión, esto es erróneo, pues, al ser varias muestras de dos elementos es insuficiente para considerarse una distribución estadística, y menos aún una distribución normal, como es la de Gauss. La curva denominada campana de Gauss da la descripción estadística estándar de un conjunto de individuos de un mismo tipo (esto es una población), en relación a sus valores de un cierto grado de libertad, continuamente variable, pero que se concentran en torno a un valor promedio más abundante. Sin embargo, no estamos ante una población estadísticamente normal o estándar, ya que la evolución de las entidades del universo es una serie de muestras de datos $95 / 5 \%$ concatenados, procedentes unos de otros anteriores. Por lo cual, la estadística que se aplica no puede ser la distribución de Gauss (reservada a poblaciones normales), como sugiere Schmitz-Moormann, ni tan siquiera otro tipo de distribución como la de Poisson, la $t$ de Student, la $F$ de Snedecor o la $c^{2}$ de Pearson, puesto que no hay un acercamiento de 
Como podemos ver en la Fig. 1, la mayor parte del universo primitivo se conserva como radiación cósmica de fondo, mientras que la materia atómica apenas resulta visible, pues su existencia se reduciría al 5\%. Cuantitativamente, este universo material se consideraría inexistente, pues más de un 99\% está compuesto por $\mathrm{H}$ y He, y los 92 elementos químicos naturales restantes constituyen menos de un $1 \%$ de la materia del universo. Tal como se ve en esta figura, la evolución a un nuevo tipo de entidad siempre tiene lugar en las regiones cuantitativamente insignificantes de la muestra anterior. Dondequiera que vemos aparecer algo nuevo en el universo en evolución, ello representa una cantidad estadísticamente insignificante en comparación con el tipo de entidad del que surgió. Así que todos los pasos evolutivos carecen cuantitativamente de importancia.

Schmitz-Moormann sentencia que el parámetro cuantitativo conduce a una alternativa de dos posibles conclusiones: o todos los tipos de entidad que configuran el universo resultan despreciables, o bien la cantidad no es una característica que diga mucho sobre lo que resulta relativamente importante en el universo. Schmitz-Moormann aboga por la segunda, pues afirma que si el argumento cuantitativo se toma en serio para explicar el significado de la evolución del universo, conduce a la absurda conclusión de que la evolución es una cuestión insignificante. Por lo tanto, concluye que realizar afirmaciones sobre la orientación de la evolución basándose puramente en la cantidad lleva a una interpretación errónea de la realidad evolutiva. Esto es, pues, lo que él llama falacia cuantitativa, análogamente a la falacia naturalista, que considera que lo natural es lo estadísticamente mayoritario.

En efecto, la distribución evolutiva está marcada por la aparición de entidades nuevas en regiones que pertenecen a acontecimientos estadísticamente muy improbables. Fijémonos en el caso de la aparición del ser humano. La probabilidad de que haya surgido el ser humano, según estos

muchas muestras a un conjunto contínuo de muestras, por decirlo de algún modo, y, por tanto, no puede tratarse como una distribución estadística, sino solo como una serie de muestras. Cfr. Schmitz-Moormann y Salmon 1997b, 35 (traducción española, 69). 
datos, sería del orden de $10^{-13}$. Ésta sería la probabilidad de que una unidad ${ }^{2}$ de radiación cósmica de fondo formara parte de un ser humano.

Contrariamente a un universo valorado en función de la cantidad, observamos la realidad de una evolución que ha ido superando todas esas improbabilidades aparentes. Sin embargo, para todas las entidades en evolución resulta esencial alguna cantidad, ya que de otro modo éstas no existirían. De ahí que Schmitz-Moormann plantea como primer presupuesto para la elaboración de sus tesis el argumento cualitativo; es decir, escoge para la interpretación del significado de la evolución del universo la cualidad, en vez de la cantidad. Esto le lleva a su segundo presupuesto, que consiste en enfatizar la importancia del ser humano (estadísticamente minoritario) frente a los microorganismos o las micropartículas (estadísticamente mayoritarios).

\section{Antropocentrismo metodológico: el ser humano como clave interpretativa del universo}

En un contexto evolutivo, la idea teológica clásica de que el mundo en su comienzo era perfecto no se puede sostener. En vez de esa perfección, la cosmología ve un caos total al inicio del universo, con ninguna entidad discernible teóricamente antes del tiempo de Planck $\left(10^{-43} \mathrm{~s}\right)$. El estado anterior no contiene ni explica el ulterior, lo cual es cierto para la evolución atómica, molecular y biológica, y especialmente para la hominización. Mirando hacia atrás se puede entender la evolución y su proceso. Pero, para entender qué significa la evolución no se ha de buscar explicaciones en los estadios inmediatamente después del Big Bang, ya que según Schmitz-Moormann no ayudan, sino que se debe considerar lo humano como la clave para entender la evolución (cfr. Schmitz-Moormann 1995, 119).

Schmitz-Moormann insiste en que para entender el pasado y su significado en el universo evolutivo, se ha de mirar atrás, comenzando por

2 Utilizamos aquí el término unidad para referirnos indistintamente a todos los elementos cósmicos: átomos, moléculas, macromoléculas, células, etc. 
nuestro conocimiento sobre la realidad presente. De acuerdo con el Principio Antrópico ${ }^{3}$, primero se debe contar con existencia humana para decir que las constantes físicas resultan importantes para la aparición de la vida, especialmente la humana. Pues, empezar con las constantes físicas identificadas en el Principio antrópico no llevaría a la realidad humana que conocemos. No podemos comprender el presente como un resultado necesario del pasado. Por otro lado, el futuro no está definido por nosotros, puesto que la evolución no llegó a un punto muerto cuando los humanos aparecieron en la Tierra. Aunque la evolución biológica continúa y, al hacerlo, promueve la diversificación de las especies, puede no ser ya el proceso principal (cfr. Schmitz-Moormann 1995, 120-121).

Schmitz-Moormann se percata de que, metodológicamente, la búsqueda de los inicios como base explicativa no resulta particularmente útil para nuestra interpretación de un mundo en evolución. Así que el relato que va desde el inicio del universo al surgimiento de la especie humana solo se puede reconstruir retrospectivamente. Además, resulta más razonable partir de lo conocido que de lo desconocido.

Ciertamente, elegir al ser humano como punto de partida para interpretar el universo como proceso evolutivo es una metodología antropocéntrica. No obstante, Schmitz-Moormann replica que esto no constituye un argumento contra el uso de esta metodología, puesto que, según la ley de Dollo ${ }^{4}$, que excluye la reversibilidad, no existe evolución retrógrada alguna.

El ser humano surgió en el proceso evolutivo como Homo sapiens. De hecho, Schmitz-Moormann aduce que los humanos nos experimentamos a nosotros mismos como centros unificados, compuestos por múltiples

3 Las observaciones de los valores de las constantes físicas, que son extremadamente sensibles, condujeron a formular el Principio Cosmológico Antrópico de Barrow y Tipler (cfr. Barrow y Tipler 1986). Una ligera desviación de uno de estos valores -en especial la constante de Coulomb y la constante gravitatoria- habría impedido la formación y la evolución del universo tal como lo conocemos y a partir del cual hemos evolucionado.

4 La ley de Dollo o ley de irreversibilidad evolutiva, propuesta en 1893 por el paleontólogo francés Louis Dollo (1857-1931), afirma que no existe evolución regresiva alguna. Es decir, es imposible que se repitan las etapas de la evolución de las especies en sentido inverso, de un descendiente a un antecesor. Esto implica que una especie extinta no puede reaparecer. La ley de Dollo constituye uno de los principios básicos de la evolución. 
partes, que nos pertenecen esencialmente. Cada ser humano incorpora la historia condensada del universo, desde el inicio de la formación de los protones hasta la formación del cerebro. En el ser humano, el proceso universal ha alcanzado un estadio en el que ha llegado a ser reflexivo.

\section{Las tesis centrales de Schmitz-Moormann sobre la evolución del universo}

Para explicar la evolución del universo desde la perspectiva cualitativa y antropocéntrica, Schmitz-Moormann propone como núcleo de sus tesis centrales los parámetros cualitativos siguientes: unión y conciencia (heredados de Teilhard, pero con puntualizaciones y matices de Schmitz-Moormann). De esas dos tesis habla en su obra básica Teología de la Creación de un mundo en evolución (cfr. Schmitz-Moormann 1997a, caps.1-4; Schmitz-Moormann y Salmon 1997b, caps. 2-5) ${ }^{5}$.

En los dos primeros capítulos de esta obra, Schmitz-Moormann desarrolla las leyes de Teilhard de Chardin sobre la metafísica de la unión: (i) el ser como plus esse que se realiza a través de la unión, y (ii) la evolución hacia la emergencia de la conciencia mediante la complejidad. Estas dos leyes teilhardianas son recogidas por Schmitz-Moormann como centrales en sus tesis evolutivas.

\subsection{Tesis $1^{a}:$ La evolución como creación por unión}

La metafísica clásica consideraba el acto de creación como una creación original (creatio) y una creación continua (conservatio). Para Schmitz-Moormann, estos términos pertenecen a un universo estático: el primero establece la creación en su perfección inicial; el segundo asegura su sostenimiento. Esta separación del acto de creación ya no es aplicable al universo en evolución. Así que no existe momento alguno en que la materia fuese creada y más tarde modificada. Según él, a causa de nuestra tendencia a interpretar la

Véase una buena síntesis del pensamiento de Karl Schmitz-Moormann y de esta obra, en particular, en Doncel 2008.

Scientia $e$ Fides $8(2) / 2020$ 
realidad dentro de un continuo temporal, caemos en la trampa de dividir el acto creador de Dios, básicamente, en un acto de tipo constitutivo y otro de tipo sustentador. Así que para Schmitz-Moormann, resulta inapropiado postular un acto especial de creación inicial complementado con un acto de creación continua, que mantenga el universo en existencia. La creación ha de considerarse como un solo acto, que es uno en la eternidad de Dios, y que se despliega en el tiempo en toda su diferencialidad (cfr. Schmitz-Moormann y Salmon 1997b, 121 [traducción española, 214]).

Schmitz-Moormann contempla la creación como un universo evolutivo que, ontológicamente, no es un mundo de seres estáticos y determinados, sino un mundo de un dinámico llegar-a-ser o realizarse (en alemán, Werden). Así que Schmitz-Moormann prefiere una nueva metafísica que contemple la dinámica del ser, ya que la concepción fijista de la tradicional metafísica aristotélico-tomista no armoniza con la evolución. Para ello, aboga por la metafísica de la unión (cfr. Casadesús 2014) de Teilhard de Chardin, que trasciende la metafísica del ser, como fundamento de sus tesis.

Con la idea de unión o unificación creciente de los elementos del universo, Schmitz-Moormann convierte nuestro concepto de entidad, elaborando el nuevo concepto de uni-totalidad (Ein-Ganzheit), totalidad unida, para desarrollar su primer parámetro cualitativo: la unión. Schmitz-Moormann aplica ampliamente el concepto de uni-totalidad a todo el proceso evolutivo. Sin embargo, mientras la aproximación a la realidad que hace Teilhard de Chardin es de abajo a arriba, ya que empieza fijándose en los fenómenos; Schmitz-Moormann por el contrario, siguiendo su segundo presupuesto, parte de los seres humanos y va buscando entidades retrospectivamente, subrayando en todas ellas cómo se comportan frente a su ambiente como unidades y totalidades cada vez más y más centradas. Así, dice en el párrafo en que introduce su nuevo concepto:

Nuestra mirada retrospectiva nos ha hecho conocer «unidad y totalidad» [«Einheit und Ganzheit»] como una característica que ha de adscribirse a todas las realidades que nos salen al encuentro en la historia de la evolución. Si bien 
esta característica señala todo cuanto ha evolucionado, hay sin embargo niveles cualitativamente diferenciables de «uni-totalidad» [«Ein-Ganzheit»] ${ }^{6}$.

De acuerdo con la unión creadora (cfr. Casadesús 2015) de Teilhard de Chardin, el proceso básico de la evolución es la unión permanente de elementos en un proceso de devenir para producir niveles más altos de ser e incluso de llegar-a-ser, sea añadiendo nuevos elementos a la totalidad, sea intensificando su modo de unión. Siguiendo esta idea de Teilhard, para Schmitz-Moormann, Dios crea las nuevas uni-totalidades mediante la unión. Los entes son creados por la unión de elementos, y los modos de unión indican niveles de ser. Véase el siguiente párrafo donde Schmitz-Moormann asume la primera ley de Teilhard como una de sus tesis:

La creación se nos hace visible como un proceso continuado bajo el aspecto básico de la unión, que produce lo nuevo, como emergiendo de los elementos que se unen, pero sin ser idéntico a ellos. Por lo tanto, podemos afirmar: a través de la unión [Dios] hace llegar a la existencia más ser. Cuanto más unido está algo en sí mismo formando una uni-totalidad, más se realiza como ser .

Schmitz-Moormann ve, pues, la metafísica de la unión como enraizada en el trascendental clásico de la unidad:

La afirmación de que la unión crea ser tiene su equivalente en uno de los trascendentales, a saber, que omne ens est unum (todo ente es uno). Esto se traduce en la metafísica de la unión por omne ens est unitum (todo ente está unido), es decir, existe como ser que se está uniendo .

6 Schmitz-Moormann 1997a, 39: “Unsere Rückschau hat uns Einheit und Ganzheit als ein Merkmal erkennen lassen, das allen Wirklichkeiten, die uns in der Geschichte der Evolution begegnen, zuzuschreiben ist. Wenn dieses Markmal auch alles, was evolviert ist, kennzeichnet, so gibt es doch qualitativ unterscheidbare Stufen der Ein-Ganzheit".

7 Schmitz-Moormann 1997a, 46-47: "Die Schöpfung wird uns unter dem Grundaspekt der Vereinigung als ein fortgehender Prozeß sichtbar, der Neues hervorbringt, das aus den Elementen emergiert, die vereint werden, das aber nicht mit den Elementen identisch ist. Wir können also feststellen: durch Vereinigung kommt Mehrheit zustande. Je mehr etwas in sich vereint ist zur Ein-Ganzheit, um so mehr Sein ist verwirklicht".

8 Ibidem, 48: "Die Feststellung, daß Vereinigung Sein schafft, hat ihr Gegenstück in einem der Universalien, nämlich daß omne ens est unum, daß alles Sein eins ist. In die Metaphysik der Vereinigung übersetzt, lautet dieser Satz omne ens est unitum, alles Sein wird geeint, das heißt existiert als geeint werdendes Sein”. 
Efectivamente, Schmitz-Moormann defiende que Dios actúa desde el interior de las cosas -como decía Teilhard-, Dios actúa de forma inmanente, Dios se revela creando mediante la unión. Por lo tanto, Dios es el Ser supremo realizado en una Unión suprema. A partir de esta premisa, Schmitz-Moormann hace la siguiente analogía: el Ser supremo como suprema unión de personas. Si Dios existe uniéndose (la Trinidad), el Ser supremo realizándose en unión tiene que ser una unión de personas (cfr. Schmitz-Moormann y Salmon 1997b, 38-49 [traducción española, 76-92]).

Aunque esta metafísica tiene su comienzo en la materia, no podemos clasificarla de monismo materialista, puesto que Schmitz-Moormann pone su pleno sentido en el espíritu. En este sentido, en el año 1973, el gran teólogo Joseph Ratzinger (nuestro actual Papa emérito) decía algo similar a lo que decimos, alabando un texto de Teilhard:

En los escritos de Teilhard de Chardin podemos leer esta observación: «Lo que distingue a un materialista de un espiritualista ya no es (como en la filosofía fijista) el hecho de que el materialista admita la transición entre una infraestructura física y una superestructura psíquica, sino tan solo el hecho de que sin razón pone el punto de equilibrio definitivo del movimiento cósmico del lado de la infraestructura, es decir, de lo que se desmorona» [cit. en Tresmontant 1961,45]. Se podrá discutir sobre detalles de este enunciado, pero en mi opinión capta con precisión lo decisivo9.

Así que tanto para Teilhard como para Schmitz-Moormann, los modos de unión remiten a niveles de ser, desde el nivel atómico al nivel humano o personal. Como decía Ratzinger, el aspecto material no es secundario, pues "el espíritu no es un producto casual de la evolución material, sino

9 Ratzinger 1973, 158: “Bei Teilhard de Chardin findet sich zu dieser Frage folgende geistvolle Bemerkung: «Was einen Materialisten von einem Spiritualisten unterscheidet, ist keineswegs mehr (wie in der fixierenden Philosophie) die Tatsache, daß einen Übergang zwischen physischer Infra-Struktur und psychischer Super-Struktur der Dinge zuläßt, sondern nur, daß er den endgültigen Gleichgewichtspunkt der kosmischen Bewegung zu Unrecht auf seiten der Infra-Struktur, das heißst des Zerfalls, setzt». Über Einzelheiten dieser Formulierung wird man sicher streiten können; das Entscheidende scheint mir hier aber treffsicher erfaßt”. 
más bien la materia constituye un momento en la historia del espíritu" ${ }^{10}$. El ser se identifica así con la unificación (Einswerdung).

Sin embargo, la unión no destruye la identidad de los elementos implicados en la uni-totalidad. La unión no es fusión sino que potencia la identidad del elemento. Así lo dice Schmitz-Moormann: "el proceso evolutivo se desarrolla a través de la unión de elementos en uni-totalidades más elevadas en las que algo nuevo llega a la existencia" ${ }^{11}$.

Para Schmitz-Moormann, la unidad constituye una característica atribuible a todas las realidades con las que nos encontramos en la historia de la evolución. No obstante, existen niveles de unidad cualitativamente distinguibles (cfr. Schmitz-Moormann y Salmon 1997b, 41 [traducción española, 81]).

En el proceso evolutivo, la unión continúa, pero los elementos que forman la unión pueden cambiar a medida que la evolución avanza. Como sabemos, en Física de partículas, la unión crea una nueva realidad con propiedades que no estaban presentes en las primeras partículas. Los elementos son una condición para que emerja la nueva realidad, aunque éstos no la contienen. Así, en Física cuántica, los quarks se unen para formar protones y neutrones. Estos dos se unen para formar núcleos, que a la vez se unen a electrones para formar átomos. Los átomos se unen para formar moléculas. Éstas formaran macromoléculas mediante la unión entre ellas, y así sucesivamente en una secuencia anidada.

$\mathrm{Al}$ nivel químico, la unión de dos átomos de $\mathrm{H}$ y un átomo de $\mathrm{O}$ forma una molécula de agua $\left(\mathrm{H}_{2} \mathrm{O}\right)$. A este nivel, se hace evidente que la nueva entidad no surge de una causa que contenía lo nuevo de una manera oculta, sino que los elementos se unen para formar una entidad única nueva, que no se encontraba contenida en los elementos. Así que los elementos (moléculas, átomos...) son un medio, no la causa, de la unidad de la totalidad.

10 Ibidem, 158: “der Geist nicht ein Zufallsprodukt materieller Entwicklungen ist, sondern daß vielmehr die Materie ein Moment an der Geschichte des Geistes bedeutet”.

11 Schmitz-Moormann y Salmon 1997b, 44: "the evolutionary process proceeds by union of elements into higher unities in which something new comes into existence”. (Véase traducción española, 86) 
Los elementos son una condición para que emerja la nueva realidad, pero éstos no la contienen.

Schmitz-Moormann pone como ejemplo a nivel biológico el caso del desarrollo del córtex visual para comprender el funcionamiento de la evolución. En este caso, el córtex visual desarrollado más tarde se hizo cargo de una manera más eficiente de las funciones del cerebro anterior, cambiando la estructura y función de este último. Luego, puede entenderse la evolución no como una adición continua de nuevos rasgos a los ya existentes, un proceso en el cual los nuevos rasgos están fundamentalmente influenciados por los existentes, sino al contrario, como un proceso que continuamente va abriéndose a nuevas posibilidades, siendo penetrado hasta la última fibra de su ser por la nueva realidad alcanzada (cfr. Schmitz-Moormann 1986, 253).

Pero, como decíamos, no en todos los niveles existe el mismo tipo de unión o cohesión. Así, las fuerzas de cohesión de los quarks dentro del protón y el neutrón (fuerzas de interacción nuclear fuerte) son diferentes a las fuerzas electromagnéticas, que atan los electrones al núcleo atómico. Asimismo, los átomos se atan formando nuevas unidades mediante diversas fuerzas electromagnéticas: iónica, covalente o de hidrógeno.

Puesto que la evolución se desarrolla mediante la unión de elementos en uni-totalidades cada vez más elevadas, Teilhard advirtió correctamente que Dios crea mediante la unión. Pero esta conclusión no se puede probar científicamente. Schmitz-Moormann subscribe la tesis de Teilhard de que a través de la unión llega a la existencia más ser. Es decir, cuanto más unido está algo en sí mismo formando una uni-totalidad, más se realiza como ser (cfr. Schmitz-Moormann y Salmon 1997b, 45 [traducción española, 87]). Pero advierte que la descripción científica de cómo se combinan estos elementos no es una explicación de la unidad interna (ontológica) que manifiestan. Así que deben existir medios de conexión (de unión) como condición necesaria para que se forme una uni-totalidad (cfr. Schmitz-Moormann y Salmon 1997b, 43 [traducción española, 83]): "Entre conocer los medios por los que la realidad individual se mantiene unida, y saber qué conforma su unidad, un tanto borrosa en los elementos simples, existe un abismo que 
se hace cada vez más evidente en los seres vivos" (cfr. Schmitz-Moormann y Salmon 1997b, 44) ${ }^{12}$.

Este factor que crea unidad y totalidad lo llamamos vida. La vida solo existe como totalidad, no como suma de partes. La vida está presente a través de la unión de las partes que cooperan como si estuviesen bajo una dirección, más que simplemente reaccionando unas con otras (cfr. Schmitz-Moormann y Salmon 1997b, 43 [traducción española, 84-85]). La vida misma evoluciona hacia seres centrados cada vez más claramente conscientes y finalmente conscientes de sí mismos.

Los animales actúan como unidades vivas centradas en sí mismas, que se relacionan con el mundo y actúan en él. Ciertamente, su mundo se limita a lo que perciben con sus sentidos, y son incapaces de superar los límites de esos sentidos. Sin embargo, vemos en nuestros ancestros biológicos la realidad de seres unificados y centrados en su propio nivel de vida.

En el reino de los mamíferos, no tenemos dificultades en ver un comportamiento centrado, unificado y totalizado. A este nivel, los individuos conocen el mundo que hay dentro de su radio de percepción y saben qué es lo que quieren hacer. Pero cuanto más retrocedemos en el pasado, más se reduce el radio de percepción y la unificación de los individuos. Como Teilhard, Schmitz-Moormann también sitúa el inicio de esa unión en la célula (cfr. Schmitz-Moormann y Salmon 1997b, 40 [traducción española, 78-79]).

El nivel más elevado de unión entre elementos se da cuando los seres humanos se unen a un nivel personal con otras personas, formando una unidad que trasciende al individuo. Teilhard de Chardin señaló que en el nivel humano la fuerza unitiva es el amor (cfr. Casadesús 2017). Su hipótesis fundamental es que la naturaleza humana es la clave para entender todo el universo, porque el ser humano es la entidad más nueva gestada por el proceso universal de la evolución, con propiedades que sobrepasan todos los otros seres que conocemos en el mundo evolutivo. Con esto, podemos definir el amor como la fuerza básica del universo evolutivo. Y ello está en

\footnotetext{
12 "This gap between knowing the means by which the individual reality is held together and what makes its unity somewhat blurred in the simple elements, becomes more evident in living beings”. (Véase traducción española, 85)
} 
armonía con el proceso evolutivo: el mismo amor no es una fuerza estática, sino que evoluciona con el universo (cfr. Schmitz-Moormann 1995, 126).

Dios, constituyente de la suprema Uni-totalidad, el supremo Ser realizándose en unión, tiene que ser una unión de personas. Y la fuerza que une es el amor. Dios existe en perfecta unión porque "Dios es amor" (1 Jn 4,8.16) (cfr. Schmitz-Moormann y Salmon 1997b, 48 [traducción española, 91]).

\subsection{Tesis $2^{\mathrm{a}}$ : La conciencia como culmen de la evolución del universo}

Schmitz-Moormann percibe la creación como un proceso no dominado por el orden, aunque con una cierta dirección general, que conduce a la conciencia. Arguye Schmitz-Moormann que Dios tiene en cuenta las leyes físicas y también el azar para crear el universo evolutivo. He ahí la intención creadora divina: que los hombres lleguen a ser capaces de encontrarse con Dios. Así, Dios permite al universo que explore por azar todas sus posibilidades y espera pacientemente a que, en el ser humano, aparezca la conciencia reflexiva. Por lo tanto, la historia del universo evolutivo, que da lugar a la aparición de nuevas realidades, no es ningún proceso ordenado: el orden aparece localmente a partir del desorden Cfr. Schmitz-Moormann y Salmon 1997b, 50-71 (traducción española, 93-128).

Schmitz-Moormann pone énfasis en que la conciencia aparece a partir de la materia, de la cual surge la vida, y con ella, la conciencia. Schmitz-Moormann defiende que la creación evolutiva tiende hacia la conciencia, pues en su punto más alto (la noosfera de Teilhard de Chardin) este proceso da lugar a la aparición de nuevas cualidades, que trascienden todas las demás cualidades de la vida, a saber, conciencia autorreflexiva, pensamiento creativo abstracto, creatividad artística... y, además, sentido.

Ciertamente, la conciencia (en sentido amplio) no es una realidad exclusivamente humana, pues en el mundo animal encontramos una amplia gama de fenómenos análogos a la conciencia ${ }^{13}$ (aunque el grado de conciencia no

13 Como observó Kandel (cfr. Kandel 1979), con solo unas pocas neuronas, el aprendizaje y la memoria resultan posibles. Los animales se adaptan activamente a su entorno y hacen uso de sus experiencias pasadas. La información sobre el entorno es almacenada internamente. Incluso el protozoo más pequeño es capaz de reaccionar activamente a la infor- 
es el mismo en todas partes), pero solo con los seres humanos el universo comienza a buscar su sentido. Además, el ser humano es la única especie cuya conciencia abarca la dimensión temporal.

Es cierto que el universo comienza con estructuras relativamente simples y evoluciona según un proceso de complejificación ${ }^{14}$ y unión. Como observamos en la Fig.1, los átomos constituyen una rareza cuando se comparan con la energía total del universo. En este universo, que permite la evolución de la vida y la conciencia, la materia realmente significativa procede de esta escasa rareza. Así, la conciencia parece ser algo insólito en el universo. Los demás planetas de nuestro sistema solar no presentan nada semejante. Pero incluso si existieran otros planetas que albergasen creaturas vivas capaces de pensar reflexivamente, esto seguiría siendo un fenómeno raro (cfr. Schmitz-Moormann y Salmon 1997b, 51 [traducción española, 95]).

La mente hace su aparición en la humanidad con la infraestructura del cerebro; pero una vez ha emergido toda realidad humana, tiene que ser explicada en términos de funciones de la mente. Así, cuando nos confrontamos con la realidad de los arquetipos (Urbild), lo que podría haber sucedido, según Schmitz-Moormann, es que la estructura preexistente de la psique animal haya sido reestructurada por la aparición de lo que llamamos mente humana, definida como pensamiento, reflexión, amor y capacidad de concebir el futuro. Por tanto, los arquetipos han llegado a ser parte de la realidad humana, y son traspasados por herencia de una generación a la siguiente en el inconsciente, como Carl Jung estableció (cfr. Schmitz-Moormann 1986, 254).

Schmitz-Moormann también se pregunta sobre la famosa y discutida relación mente-cerebro. La cuestión existencial de los seres humanos es ¿quiénes somos los que somos capaces de pensar, querer y rezar?

mación que obtiene del exterior. Al menos en los animales encontramos indicios de conciencia, entendida ésta como exploración activa del entorno. Obviamente, no llamamos conciencia a esta habilidad, pero tiene en común con ella que la información del exterior es internalizada, y se reacciona a esta información a fin de mantener la propia existencia. Cfr. Schmitz-Moormann y Salmon 1997b, 54-59 (traducción española, 100-109).

14 Teilhard de Chardin introdujo por primera vez el concepto de complejidad para medir el nivel de evolución e indicó que existe una estrecha relación entre el crecimiento de complejidad y el crecimiento de centricidad o conciencia. Cfr. Casadesús 2014. 
Schmitz-Moormann opta por que el cerebro es la herramienta funcional dominada por la mente, ya que el cerebro humano está constantemente influenciado por información esencialmente inmaterial, que produce cambios materiales en el cerebro. Esta información toma parte en nuestra composición física; véase si no el caso del lenguaje.

Aunque el problema mente-cuerpo aún no ha sido solucionado totalmente, podemos decir que la mente resulta altamente efectiva por el hecho de ser reflexivamente consciente. La conciencia hace posible el desarrollo y la utilización de conceptos abstractos que se pueden manejar al tiempo que la realidad concreta se halla ausente. Los seres humanos conocen el mundo que les rodea, y utilizan este conocimiento de manera efectiva para cambiar el entorno. Así, la conciencia no es solo saber acerca de uno mismo, sino entender el mundo que nos rodea (cfr. Schmitz-Moormann y Salmon 1997b, 54-55 [traducción española, 101-102]).

La pequeña masa del cerebro humano, siendo éste la infraestructura visible de la mente humana, puede llegar mucho más allá del universo entero. Siguiendo a Teilhard de Chardin, que consideraba el cerebro como la forma de la materia en la que su espiritualidad llega a ser más evidente, para Schmitz-Moormann, el ser humano, en cuanto a su dimensión espiritual, es capaz de superar la realidad cuantitativa de este universo (cfr. Schmitz-Moormann y Salmon 1997b, 52 [traducción española, 97]). El ser humano es el único ser que todo el mundo conoce desde la propia conciencia y, además, el único que conoce los motivos de sus acciones.

Sin embargo, al contrario de Teilhard, para Schmitz-Moormann (cfr. Schmitz-Moormann y Salmon 1997b, 57 [traducción española, 106]), la conciencia parece desaparecer completamente por debajo de la biosfera. Nosotros estamos de acuerdo con eso. Al nivel de las moléculas y los átomos, la conciencia ya no resulta detectable. Con todo, los átomos y moléculas constituyen la condición necesaria para que la conciencia aparezca en el universo. La conciencia aparece, pues, como una realidad en los seres humanos y disminuye a medida que retrocedemos en la historia de la evolución.

Si nos fijamos en las realidades emergentes del universo, que representan solo cantidades mínimas, podemos apreciar una cierta orientación 
general. La afirmación de que no existe orientación alguna en el proceso evolutivo está basada en un argumento cuantitativo, que no sirve para describir orientación en la evolución. Como explicamos en §1, la evolución más significativa ha tenido lugar dentro de una zona estadísticamente improbable. La media estadística no nos aporta información acerca de la orientación de la evolución. Ésta sigue el camino de lo improbable, y resulta razonable considerar la historia de la conciencia emergente como la más significativa del universo (cfr. Schmitz-Moormann y Salmon 1997b, 58-59 [traducción española, 108]).

Aunque Schmitz-Moormann aboga por una orientación general del universo, puesto que la evolución da lugar a posibilidades que permiten la emergencia de formas más elevadas de conciencia, ya que con una mirada retrospectiva se puede ver una importante y continua dirección de la conciencia emergente; sin embargo, señala que no existe una necesidad inherente de que ese proceso tome la dirección que toma. De este modo, Schmitz-Moormann no cae en el determinismo. En este punto, Schmitz-Moormann no está de acuerdo con Teilhard de Chardin, quien identificaba la ortogénesis con la evolución (cfr. Casadesús 2014). Así, para Schmitz-Moormann, la orientación del proceso evolutivo hacia la conciencia no se puede identificar con la ortogénesis determinista clásica, ya que la ortogénesis es un proceso lineal, deducible y predecible.

Algunos físicos presuponen que el mundo es perfectamente explicable por su inicio. Pero, puesto que, en la práctica, muchos sistemas dinámicos son caóticos (los vitales lo son todos, y los humanos aún más), el caos determinista no es un sistema perfectamente precisable en sus condiciones iniciales, por más que lo sea en sus ecuaciones. Así que el futuro prácticamente no está determinado, aunque se conozca que tiene un número limitado de resultados. En los niveles más bajos de la evolución, estos resultados parecen suceder por azar, pero a nivel humano están en gran medida en manos de los agentes responsables, debido al libre albedrío. Así que en el nivel humano, la evolución es fomentada por las acciones y decisiones humanas (cfr. Schmitz-Moormann 1995, 127). 
Actualmente, está muy extendida la idea de que el proceso de la evolución, en todos sus estadios, es un proceso de complejificación. En un momento determinado, este proceso emerge en la vida. La vida no constituye una propiedad de algo; es más bien una nueva realidad en una infraestructura ya altamente compleja, que emerge a partir de un proceso evolutivo a través de niveles crecientes de complejidad y, al mismo tiempo, de unión.

En cambio, la evolución da lugar a probabilidades que permiten, en sus extremos, la emergencia de formas más elevadas de conciencia. Los indicios más primitivos de conciencia se pueden describir como una totalidad que internaliza la experiencia de su entorno -es decir, que aprende- y reacciona activamente como totalidad a ese entorno (cfr. Schmitz-Moormann y Salmon 1997b, 58 [traducción española, 108]).

\section{Conclusión}

El obejtivo de Karl Schmitz-Moormann fue descubrir las huellas de Dios creador en el universo. Para ello, Schmitz-Moormann comienza denunciando lo que él denomina falacia cuantitativa, que hemos presentado en $\S 1$, que significa que lo más importante para la evolución del universo ha sido lo cuantitativamente más abundante. Además, también defiende un antropocentrismo metodológico (explicado en §2) que, ciertamente, puede relacionarse con el Principio antrópico, puesto que, para Schmitz-Moormann, el universo solo puede entenderse a partir del ser humano.

Partiendo de estos dos presupuestos, hemos desarrollado en este artículo las primeras dos tesis evolutivas de Schmitz-Moormann, directamente influenciadas por Teilhard de Chardin. De ahí que Schmitz-Moormann ve como aspectos básicos de la evolución global: por un lado, la unidad y la creación de unidad, formulando el nuevo concepto de uni-totalidad o totalidad unida; y, por otro lado, el surgimiento de la conciencia y su crecimiento.

Para demostrar estas dos tesis evolutivas, Schmitz-Moormann realiza una panorámica retrospectiva desde la actualidad hasta el Big Bang; dicho de otro modo, parte del ser humano hasta llegar a las partículas subatómicas. Con ello, pensamos que muestra que todos los entes que han evolucionado 
tienen como característica fundamental ser totalidades unidas (o Einheit und Ganzheit), que él denomina con el neologismo uni-totalidad (Ein-Ganzheit). Además, Schmitz-Moormann añade que esas uni-totalidades han ido enriqueciéndose -como diría Teilhard de Chardin- a lo largo de la historia de la evolución por un crecimiento de centramiento, centricidad o conciencia (centrement, centrité o centréité, para Teilhard) desde los elementos más ínfimos hasta el ser humano.

En definitiva, hemos presentado aquí cómo Schmitz-Moormann expone sus tesis evolutivas, basadas en el proceso de llegar-a-ser como un proceso de unión y de crecimiento de conciencia, desarrollando la metafísica de Teilhard de Chardin, precisándola e enriqueciéndola con matices propios.

\section{Bibliografía}

Barrow, John, y Frank J. Tipler. 1986. The Anthropic Cosmological Principle. Oxford: Clarendon.

Casadesús, Ricard. 2014. "L'évolution comme métaphysique de l'union chez Teilhard de Chardin.” Revue des Questions Scientifiques 185: 373-398.

Casadesús, Ricard. 2015. “¿Creare est unire? Esbozo de la metafísica de la unión de Teilhard de Chardin.” Scientia et Fides 3: 137-160.

Casadesús, Ricard. 2017. "El amor como fuerza conductora del desarrollo evolutivo en Teilhard de Chardin.” Quaerentibus. Teología y Ciencias 8: 213-224.

Doncel, Manuel G. 2008. “Teología de la evolución (II): La llamada creadora trinitaria. Karl Schmitz-Moormann, 1997.” Pensamiento. Revista de investigación e información filosófica 64: 783-814.

Kandel, Eric R. 1979. "Small systems of neurons." Scientific American 241: 66-78.

Ratzinger, Joseph. 1973. Dogma und Verkündigung. Munich y Friburgo de Brisgovia: Erich Wewel Verlag.

Schmitz-Moormann, Karl. 1986. "Philosophical and Theological reflections on recent neurobiological discoveries.” Zygon: Journal of Religion and Science 21: 249-257.

Schmitz-Moormann, Karl. 1995. “The future of Teilhardian Theology.” Zygon: Journal of Religion and Science 30: 117-129.

Schmitz-Moormann, Karl. 1997a. Materie-Leben-Geist: Evolution als Schöpfung Gottes. Mainz: Mathias Grünewald Verlag. 
Schmitz-Moormann, Karl, y James F. Salmon. 1997b. Theology of Creation in an evolutionary world. Cleveland: The Pilgrim Press. Traducción española: 2005. Teología de la creación de un mundo en evolución. Estella: Verbo Divino.

Teilhard de Chardin, Marie.-Joseph P. 1971. Euvre Scientifique. Edición de Karl Schmitz-Moormann y Nicole Schmitz-Moormann. Aquisgrán - Olten - Friburgo: Walter Verlag, vols. I-XI.

Teilhard de Chardin, Marie.-Joseph P. 1974-1977. Journal. 26 août 1915-25 fevrier 1920. Edición de Karl Schmitz-Moormann y Nicole Schmitz-Moormann. Olten y Friburgo: Walter Verlag, vols. I-III.

Tresmontant, Claude. 1961. Einführung in das Denken Teilhard de Chardins. Friburgo y Munich: Karl Alber. 